\title{
Set Systems with Restricted $t$-wise Intersections modulo Prime Powers
}

\author{
Rudy X. J. Liu \\ Department of Mathematics, Pearl River College \\ Tianjin University of Finance \& Economics, Tianjin, 301811, P. R. China \\ xiaojiethink@yahoo.com.cn \\ Submitted: Jan 4, 2009; Accepted: May 24, 2009; Published: Jun 5, 2009 \\ Mathematics Subject Classifications: 05D05
}

\begin{abstract}
We give a polynomial upper bound on the size of set systems with restricted $t$-wise intersections modulo prime powers. Let $t \geq 2$. Let $p$ be a prime and $q=p^{\alpha}$ be a prime power. Let $\mathcal{L}=\left\{l_{1}, l_{2}, \ldots, l_{s}\right\}$ be a subset of $\{0,1,2, \ldots, q-1\}$. If $\mathcal{F}$ is a family of subsets of an $n$ element set $X$ such that $\left|F_{1} \cap \cdots \cap F_{t}\right|(\bmod q) \in \mathcal{L}$ for any collection of $t$ distinct sets from $\mathcal{F}$ and $|F|(\bmod q) \notin \mathcal{L}$ for every $F \in \mathcal{F}$, then

$$
|\mathcal{F}| \leq \frac{t(t-1)}{2} \sum_{i=0}^{2^{s-1}}\left(\begin{array}{l}
n \\
i
\end{array}\right) .
$$

Our result extends a theorem of Babai, Frankl, Kutin, and Štefankovič, who studied the 2-wise case for prime power moduli, and also complements a result of Grolmusz that no polynomial upper bound holds for non-prime-power composite moduli.
\end{abstract}

\section{Introduction}

We are interested in set systems with restricted $t$-wise intersections modulo prime powers. Let $X$ denote a set of $n$ elements and $\mathcal{F}$ be a family of subsets of $X$. Let $p$ be a prime and $q=p^{\alpha}$ be a prime power. Let $\mathcal{L}$ be a subset of $\{0,1,2, \ldots, q-1\}$ of size $s$. For an integer $t \geq 2$, a family $\mathcal{F}$ is called $t$-wise $q$-modular $\mathcal{L}$-intersecting if $\left|F_{1} \cap \cdots \cap F_{t}\right|(\bmod q) \in \mathcal{L}$ for any collection of $t$ distinct sets from $\mathcal{F}$ and $|F|(\bmod q) \notin \mathcal{L}$ for every $F \in \mathcal{F}$. It is called $q$-modular $\mathcal{L}$-intersecting for simplicity when $t=2$. Note that, the same definition is also used when $q$ is not a prime power.

In 2001, Babai, Frankl, Kutin, and Štefankovič proved the size of a $p^{\alpha}$-modular $\mathcal{L}$ intersecting family is polynomial bounded as a function of $n$. 
Theorem 1 (Babai et al. [1]) If $\mathcal{F}$ is a $p^{\alpha}$-modular $\mathcal{L}$-intersecting family of subsets of $X$, then

$$
|\mathcal{F}| \leq\left(\begin{array}{c}
n \\
2^{s-1}
\end{array}\right)+\left(\begin{array}{c}
n \\
2^{s-1}-1
\end{array}\right)+\cdots+\left(\begin{array}{c}
n \\
0
\end{array}\right) .
$$

When $q=p$, Grolmusz [5] proved the following result in 2002 .

Theorem 2 (Grolmusz [5]) If $\mathcal{F}$ is a t-wise p-modular $\mathcal{L}$-intersecting family of subsets of $X$, then

$$
|\mathcal{F}| \leq(t-1) \sum_{i=0}^{s}\left(\begin{array}{l}
n \\
i
\end{array}\right) .
$$

When $t=2$, it is a modular version of the celebrated Frankl-Wilson Theorem. Grolmusz and Sudakov [6] gave another proof of this bound using multilinear polynomials. Recently, Cao, Hwang and West [2] improved the above bound by replacing $\left(\begin{array}{l}n \\ i\end{array}\right)$ with $\left(\begin{array}{c}n-1 \\ i\end{array}\right)$ in the sum.

In the same paper [5], Grolmusz also showed that Theorem 2 does not generalize to non-prime-power composite moduli. In particular for any $t \geq 2, q=6$ and $\mathcal{L}=\{1, \ldots, 5\}$, there exists a $t$-wise 6 -modular $\mathcal{L}$-intersecting family of $X$ of superpolynomial size in $n$, see Theorem 11 in [5] for detail.

In this paper, we will fill the gap between Theorem 2 (prime moduli) and Grolmusz's result (non-prime-power composite moduli, Theorem 11 in [5]) by proving a polynomial upper bound on the size of the $t$-wise $p^{\alpha}$-modular $\mathcal{L}$-intersecting families for any $t \geq 2$.

Theorem 3 If $\mathcal{F}$ is a $t$-wise $p^{\alpha}$-modular $\mathcal{L}$-intersecting family of subsets of $X$, then

$$
|\mathcal{F}| \leq \frac{t(t-1)}{2} \sum_{i=0}^{2^{s-1}}\left(\begin{array}{c}
n \\
i
\end{array}\right) .
$$

Clearly, the special case $t=2$ of Theorem 3 corresponds to Theorem 1 .

\section{The Proof}

In this section, let $q=p^{\alpha}$ be a prime power and we will give a proof of Theorem 3, which is motivated by the methods used in [1] and [3].

First we need the following Frankl-Wilson-type result for pairs of families of sets with restricted intersection modulo prime power, which is a slight generalization of Theorem 1.

Lemma 1 Let $A_{1}, \ldots, A_{m}$ and $B_{1}, \ldots, B_{m}$ be two families of subsets of $X$ such that $\left|A_{i} \cap B_{i}\right|(\bmod q) \notin \mathcal{L}$ for all $1 \leq i \leq m$ and $\left|A_{i} \cap B_{j}\right|(\bmod q) \in \mathcal{L}$ for $i \neq j$. Then

$$
m \leq\left(\begin{array}{c}
n \\
2^{s-1}
\end{array}\right)+\left(\begin{array}{c}
n \\
2^{s-1}-1
\end{array}\right)+\cdots+\left(\begin{array}{l}
n \\
0
\end{array}\right) .
$$


Note that Theorem 1 is a special case of Lemma 1 when $A_{i}=B_{i}$ for $1 \leq i \leq m$. The proof of this lemma follows from the proof of Lemma 3.1 in [1], and we refer the reader there for details.

Proof of Theorem 3 Let us apply induction on $t$. When $t=2$, it has been proved by Theorem 1. Now assume that $t>2$ and the assertion is true for $t=k$, we will prove that it also holds for $t=k+1$.

Let $\mathcal{F}=\left\{F_{1}, \ldots, F_{m}\right\}$ be a $(k+1)$-wise $q$-modular $\mathcal{L}$-intersecting family of subsets of $X$. To prove the statement, we partition $\mathcal{F}$ into three families of sets $\mathcal{A}, \mathcal{F}_{1}$ and $\mathcal{F}_{2}$ with the following properties: there exists a family of sets $\mathcal{B}$ such that the pair $(\mathcal{A}, \mathcal{B})$ satisfies the condition of Lemma $1,\left|\mathcal{F}_{1}\right|=(k-1)|\mathcal{A}|$ and the family $\mathcal{F}_{2}$ is $k$-wise $q$-modular $\mathcal{L}$ intersecting. To do this we repeat the following procedure. For every $0 \leq r \leq|\mathcal{F}|-1$, suppose that after step $r$ we have already constructed families of sets $\mathcal{A}=\left\{A_{1}, \ldots, A_{i}\right\}$, $\mathcal{B}=\left\{B_{1}, \ldots, B_{i}\right\}, \mathcal{F}_{1}$ and $\mathcal{F}_{2}=\left\{D_{1}, \ldots, D_{j}\right\}$ such that $\left|\mathcal{F}_{1}\right|=(k-1) i$. Consider three possible cases.

Case 1: If $F_{r+1} \in \mathcal{F}_{1}$, then proceed to the next step.

Case 2: If there are indices $r+1<r_{1}<\cdots<r_{k-1}$ such that $F_{r_{i}} \notin \mathcal{F}_{1}$ for all $1 \leq i \leq k-1$ and $\left|F_{r+1} \cap F_{r_{1}} \cap \cdots \cap F_{r_{k-1}}\right|(\bmod q) \notin \mathcal{L}$, then define $A_{i+1}=F_{r+1}$, $B_{i+1}=F_{r+1} \cap F_{r_{1}} \cap \cdots \cap F_{r_{k-1}}$. Let $\mathcal{F}_{1}=\mathcal{F}_{1} \cup\left\{F_{r_{1}}, \cdots, F_{r_{k-1}}\right\}$ and proceed to the next step.

Case 3: Suppose that $\left|F_{r+1} \cap F_{r_{1}} \cap \cdots \cap F_{r_{k-1}}\right|(\bmod q) \in \mathcal{L}$ for every set of indices $r+1<r_{1}<\cdots<r_{k-1}$ with $F_{r_{i}} \notin \mathcal{F}_{1}$ for all $1 \leq i \leq k-1$. In this case define $D_{j+1}=F_{r+1}$ and continue. Clearly, by construction, $\mathcal{F}_{2}$ is a $k$-wise $q$-modular $\mathcal{L}$-intersecting family.

Let $\mathcal{A}=\left\{A_{1}, \ldots, A_{h}\right\}, \mathcal{B}=\left\{B_{1}, \ldots, B_{h}\right\}, \mathcal{F}_{1}$ and $\mathcal{F}_{2}$ be the set systems obtained in the end of our procedure. Note that, by definition, $\left|A_{i} \cap B_{i}\right|(\bmod q) \notin \mathcal{L}$ for $1 \leq i \leq h$ but $\left|A_{i} \cap B_{j}\right|(\bmod q) \in \mathcal{L}$ for $i \neq j$, since this is a size of intersection of $k+1$ distinct members of $\mathcal{F}$.

Now we can apply Lemma 1 to bound the size of $\mathcal{A}$. Since $\mathcal{F}=\mathcal{A} \cup \mathcal{F}_{1} \cup \mathcal{F}_{2}$ and $\left|\mathcal{F}_{1}\right|=(k-1)|\mathcal{A}|$, by the induction hypothesis we obtain that

$$
\begin{aligned}
|\mathcal{F}| & \leq|\mathcal{A}|+\left|\mathcal{F}_{1}\right|+\left|\mathcal{F}_{2}\right|=k|\mathcal{A}|+\left|\mathcal{F}_{2}\right| \\
& \leq k \sum_{i=0}^{2^{s-1}}\left(\begin{array}{c}
n \\
i
\end{array}\right)+\frac{k(k-1)}{2} \sum_{i=0}^{2^{s-1}}\left(\begin{array}{c}
n \\
i
\end{array}\right) \\
& =\frac{k(k+1)}{2} \sum_{i=0}^{2^{s-1}}\left(\begin{array}{c}
n \\
i
\end{array}\right) .
\end{aligned}
$$

This completes the proof of the theorem.

\section{Concluding Remarks}

The main point we make is that our bound in Theorem 3 implies a polynomial upper bound in $n$ for the $t$-wise $p^{\alpha}$-modular $\mathcal{L}$-intersecting families with $t \geq 3$. For the special 
case of prime power moduli $q$ and $s=q-1$, the bound in Theorem 3 can be improved.

Theorem 4 (Grolmusz and Sudakov [6]) Let $t \geq 2$ and $r$ be integers. If $\mathcal{F}$ is a family of subsets of $X$ such that $|F|(\bmod q)=r$ for each $F \in \mathcal{F}$ and $\left|F_{1} \cap \cdots \cap F_{t}\right|(\bmod q) \neq r$ for any collection of $t$ distinct sets from $\mathcal{F}$, then

$$
|\mathcal{F}| \leq(t-1) \sum_{i=0}^{q-1}\left(\begin{array}{l}
n \\
i
\end{array}\right) .
$$

Still it would be interesting to obtain improved upper bound for our results.

Acknowledgments. I would like to thank my research supervisor, Professor Jiuqiang Liu for his support, especially during the last 2 years. This work was partially done when I was a student in Center for Combinatorics in Nankai University. I would also like to thank an anonymous referee for some helpful suggestions.

\section{References}

[1] L. Babai, P. Frankl, S. Kutin, and D. Štefankovič, Set systems with restricted intersections modulo prime powers, J. Combinatorial Theory, Ser. A, 95 (2001), 39-73.

[2] Weiting Cao, Kyung-Won Hwang, and Douglas B. West, Improved bounds on families under $k$-wise set-intersection constraints, Graphs and Combinatorics, 23 (2007), 381-386.

[3] Z. Füredi and B. Sudakov, Extremal set systems with restricted $k$-wise intersections, $J$. Combinatorial Theory, Ser. A, 105 (2004), 143-159.

[4] Vince Grolmusz, Superpolynomial size set-systems with restricted intersections mod 6 and explicit Ramsey graphs, Combinatorica, 20, No.1, (2000), 71-86.

[5] Vince Grolmusz, Set-systems with restricted multiple intersections, Electronic J. Combinatorics, 9 (2002), R8.

[6] Vince Grolmusz and Benny Sudakov, On $k$-wise set-intersections and $k$-wise hamming distances, J. Combinatorial Theory, Ser. A, 99 (2002), 180-190. 